\title{
HHV-6A in vitro infection of thyrocytes and $T$ cells alters the expression of miRNA associated to autoimmune thyroiditis
}

\author{
Elisabetta Caselli ${ }^{1 *}$ D, Maria D'Accolti ${ }^{1}$, Irene Soffritti ${ }^{1}$, Maria Chiara Zatelli ${ }^{2}$, Roberta Rossi ${ }^{3}$, Ettore degli Uberti ${ }^{2}$ \\ and Dario Di Luca ${ }^{1}$
}

\begin{abstract}
Background: Human herpesviruses have been hypothesized as environmental triggers in the development of autoimmune thyroid diseases (AITD), and in particular active human herpesvirus 6A (HHV-6A) infection was detected in thyrocytes of Hashimoto's thyroiditis (HT) patients, who also show specific anti-viral immune responses. On the other hand, AITD patients display modulation of specific miRNAs in thyroid tissue and blood. We wanted to ascertain whether HHV-6A infection might be correlated to the miRNA dysregulation observed in AITD.

Methods: Human thyroid and T-cell lines were infected in vitro with HHV-6A,-6B or -7 , and analysed for miRNAs expression, either by microarray or by specific RT-PCR assays detecting miRNAs associated with AITD in vivo.

Results: HHV-6A infection, but not $-6 \mathrm{~B}$ or -7 infections, induced a decrease in miR-155_2 expression and an increase in miR-1238 expression in thyrocytes, as well as an increase in the expression levels of several autoimmunity-associated miRNAs in T lymphocytes, including miR-16_1, miR34a, miR-130a, miR-143_1, miR-202, miR-301b, miR-302c, miR-449b, miR-451_1, and miR-1238_2.

Conclusions: HHV-6A infection modulates miRNAs expression in the cell types involved in the development of AITD. Notably, our in vitro findings correlate with what observed in AITD patients, further supporting the association between HHV-6A infection and AITD development. Moreover, these effects are 6A-specific, emphasizing the differences between the two HHV-6 virus species, and suggesting diverse virus mechanisms of action and therapeutic approaches.
\end{abstract}

Keywords: HHV-6, miRNA, Autoimmune thyroiditis

\section{Background}

Autoimmune thyroid diseases (AITD) are very common thyroid disorders, showing increased prevalence in recent years [1]. In addition to genetic background, several viruses, including herpesviruses, have been suggested as possible environmental causes, but conclusive data are still lacking. In particular, we and others reported a high prevalence of active human herpesvirus 6A (HHV-6A) infection in thyrocytes of Hashimoto's thyroiditis (HT) patients [2, 3], who also display specific cellular and humoral anti-viral responses, increased NK killing of

\footnotetext{
* Correspondence: elisabetta.caselli@unife.it; csb@unife.it

${ }^{1}$ Department of Medical Sciences, Section of Microbiology and Medical

Genetics, University of Ferrara, via L. Borsari 46, 44121 Ferrara, Italy

Full list of author information is available at the end of the article
}

infected thyroid cells [3], and increased CD3CD56 ${ }^{\text {bright }} \mathrm{CD} 16^{-} \mathrm{NK}$ cells, whose activation significantly correlates with plasma levels of anti-thyroid peroxidase (TPO) and anti-thyroglobulin (Tg) autoantibodies [4].

HHV-6 is a ubiquitous virus including two distinct viral species, HHV-6A and HHV-6B, displaying different cellular tropism and receptors usage [5-8]. Although originally considered lymphotropic, the in vivo tropism of both viruses is considerably broader, including macrophages, endothelial cells, salivary glands, and brain [5]. HHV-6B is associated with childhood exanthema subitum, whereas HHV-6A is mostly adult-associated, and has been correlated to several chronic autoimmune inflammatory diseases, including AITD $[5,9]$. 
On the other hand, some microRNAs (miRNA) have been found dysregulated in AITD patients and implicated in the disease development. In particular, miR-155_2 has been found decreased and miR-200al increased in HT thyroid tissue compared with healthy controls [10], whereas miR-16, miR-22, miR-375 and miR-451 were found increased in serum of HT and Grave's Disease (GD) patients, compared with controls [11].

Some miRNAs function has been associated with the control of innate and adaptive immune responses [12], but further work is needed to understand the role of miRNAs and their potential as disease markers and/or therapeutic targets in autoimmune thyroiditis. Furthermore, no data are available on the potential ability of HHV-6 to induce miRNAs modulation in cells involved in the disease development (i.e. thyrocytes and lymphocytes).

Based on these observations, the present study was addressed to ascertain whether HHV-6A infection might induce modulation of those miRNAs associated in vivo with AITD, and particularly with HT.

\section{Methods}

\section{In vitro virus infection}

The human thyroid Nthy-ori3-1 and Jurkat $\mathrm{T}$ cell lines were used for in vitro infection experiments. The cells were propagated and seeded at optimal density [3], then infected with HHV-6A, $-6 \mathrm{~B}$ or -7 cell-free virus inocula, at a m.o.i. of 10 genome equivalents per cell, as previously detailed [3]. Control cells were infected with the correspondent UV-inactivated viruses, and virus infection was monitored by analyzing virus presence and transcription in infected cells, respectively by real time quantitative PCR (qPCR) and qPCR after retrotranscription (RT-qPCR), amplifying the U42 gene of all viruses, as previously described [3].

\section{miRNA analysis}

The mature miRNA fraction was extracted from infected or control cells by the miRNeasy mini kit (Qiagen, Hilden, Germany), and retro-transcribed as indicated by the manufacturer (miScript RT kit; Qiagen, Hilden, Germany). miRNA fraction was then analysed by a microarray detecting a panel of 84 microRNAs specifically associated to inflammation and autoimmunity (Qiagen, Hilden, Germany), and by specific real time quantitative PCR (qPCR) assays for the following miRNAs: miR-16-1_1, miR-16_2, miR-22, miR-34a, miR143_1, miR-146a_1, miR-155_1, miR-155_2, miR-181a_1, miR-181a_2, miR-200a_1, miR-200a_2, miR-375, miR451miR-1238_2. Two assays detecting constitutively expressed cellular miRNAs (miR-RTC and miRSNORD11) were also used as controls.

\section{Statistical analysis}

Statistical analysis of collected data was performed by Student's $t$ test.

\section{Results}

Herpesvirus infection in human thyroid and T-cell lines Prior to study miRNA expression, the infection efficiency of the viruses used for the experiments was checked by qPCR in both thyroid Nthy-ori3-1 and lymphoid Jurkat cells, showing that all virus types (HHV-6A, HHV-6B and HHV-7) were equally entering and replicating in both Nthy-ori3-1 and Jurkat $\mathrm{T}$ cells (Fig. 1), confirming what previously published $[3,5,13]$.

\section{miRNA expression in infected thyroid and T-cell lines}

miRNA expression in infected cells was analysed at 0,7 , 24, 48, $72 \mathrm{~h}$ post infection (h.p.i.) and 7 days post infection (d.p.i.). Briefly, the mature miRNA fraction was extracted from infected and control cells, retro-transcribed and analysed by two different qPCR methods. At 48 h.p.i., extracted miRNAs were analysed by a qPCR microarray detecting a panel of 84 microRNAs specifically associated to inflammation and autoimmunity; at all times post infection miRNA fraction was instead analysed by specific qPCR assays detecting the following miRNAs: miR-16-1_1, miR-16_2, miR-22, miR-34a, miR143_1, miR-146a_1, miR-155_1, miR-155_2, miR-181a_1, miR-181a_2, miR-200a_1, miR-200a_2, miR-375, miR451miR-1238_2; miR-RTC and miR-SNORD11 were used as internal controls.

As shown in Fig. 2a, in vitro HHV-6A infection of Nthy-ori3-1 thyroid cells did not induce relevant alterations in the expression of over 80 cellular miRNAs associated to inflammation and autoimmunity. In fact, as judged by microarray results at 48 h.p.i., miRNA expression was only modestly modulated (less than 3-fold changes), with most of miRNA resulting slightly downregulated (2.2 fold for miR-181d, miR-302c, miR-372, miR-520e and miR-875; 2.4 fold for miR-520d). Conversely, infection of $\mathrm{T}$ lymphoid cells resulted in a very different picture at 48 h.p.i. (Fig. 2b). In fact, HHV-6A infection (but not HHV-6B or HHV-7 infection) induced a remarkable increase in several autoimmunity-related miRNAs, namely: miR-16, miR-34a, miR-130a, miR-202, miR-301b, miR-302c, and miR-449b.

Contrarily to what observed my microarray analysis, when analyzing individual expression of AITDassociated miRNAs in thyroid cells, results showed that HHV-6A infection, but not HHV-6B or HHV-7 infection, induced in Nthy-ori3-1 thyroid cells an up to 6fold decrease in miR-155_2 and an up to 7-fold increase in miR-1238_2 levels (Fig. 3a). Virus-induced miRNA modulations were already detectable at 7 h.p.i., persisted 


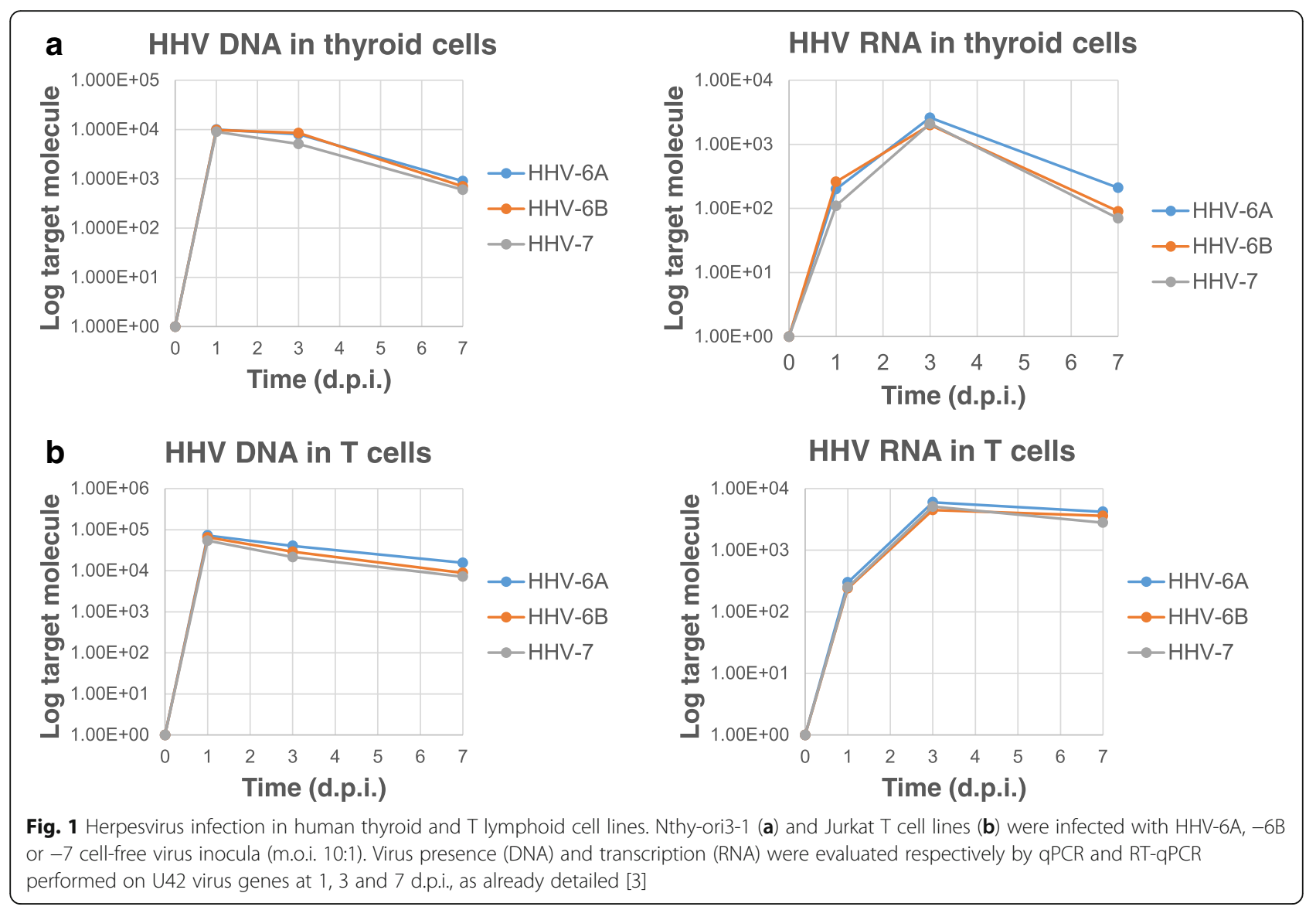

till 7 d.p.i., and were statistically significant at all times post infection $(p<0.001$ and $p<0.01)$.

In parallel, the individual analysis of AITD-related miRNAs in Jurkat $\mathrm{T}$ cells evidenced, in HHV-6A infected cells only, statistically significant increases in the expression of miR-16_1 (13-fold peak at 7 h.p.i.), miR143_1 (28-fold peak at 48 h.p.i.), miR-451_1 (36-fold peak at 48 h.p.i. $)$ and miR-1238_2 $\left(\sim 10^{4}\right.$-fold increase at 48 and 72 h.p.i.) $(p<0.0001$ and $p<0.001)$ (Fig. 3b). On the other hand, similarly to what observed in thyroid cells, no significant alteration in miRNA expression were observed in HHV-6B or HHV-7 infected T cells, except for a slight increase in miR-155_2 levels in HHV-7 infected cells at 7 h.p.i.

\section{Discussion}

Our data show for the first time that in vitro HHV-6A infection of human cells of thyroid and $\mathrm{T}$ lymphoid origin (namely Nthy-ori3-1 and Jurkat cell lines) induces modulation of miRNAs considered markers of AITD in vivo. Notably, the virus-induced alterations correlate with what observed in vivo, as AITD patients, particularly those affected by $\mathrm{HT}$, have decreased miR$155 \_2$ in thyroid tissue [10], and increased miR-16 in serum [11].

Due to their emerging role as important regulators of immune function [14], the virus-induced dysregulation of these miRNAs might have important consequences in the development of autoimmunity. In particular, miR155 is a pleiotropic modulator of innate and adaptive immunity [15-17], and might promote autoimmune inflammation [18]. Beside its role as tumor-suppressor [19], miR-16 was recently shown to affect NK function by repressing IFN $\gamma$ expression [20], and to promote M1 pro-inflammatory type macrophage polarization, affecting $\mathrm{T}$ cell activation [21]. No data are instead available on miR-1238 potential functions in immunity, as it was so far recognized only as a tumor-suppressor factor [22]. However, it was identified as a marker of AITD [23], together with miR-143_1, and the strong increase observed in both thyroid and lymphoid HHV-6A infected cells suggest a potential role of miR-1238 in the modulation of immune response, and deserves further studies.

Notably, significant alterations were detected only in HHV-6A infected cells, and not in HHV-6B or HHV-7 


\section{Thyroid cells}

a

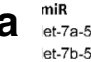
et-7b-5p
et-7c-5p
et-7d-5p et-7e-5p et-7f-5p et-7g-5p et-7i-5p miR-15a-5p miR-15b-5p MiR-16-5p $\operatorname{miR}-17-5 p$ MiR-19a-3p niR-19b-3p MiR-20a-5p MiR-20b-5p miR-23a miR-23a-3p MiR-23b-3p miR-29a-3p $\operatorname{miR}-29 \mathrm{~b}-3 \mathrm{p}$ MiR-29c-3p miR-30a- $5 p$
niR-30b-5p MiR-30b-5p
miR-30c-5p miR-30c-5p miR-30d-5p miR-30e-5p miR-34a-5p

miR-34c-5p
miR-39-3p

miR-39-3p

miR-93-5p
miR-98-5p

miR-101-3p

niR-106b-5p

miR-125a-5p

miR-125b-5

miR-12B-3p

miR-130b-3p

miR-144-3p

miR-145-5p

niR-181a-5p
niR-181b-5p

miR-181c-5p

miR-181d-5p

miR-186-5p

MiR-195-5p

niR-202-3p

niR-211-5p

min-300

MiR-301a-3p

miR-301b-3p
miR-302a-3p

MiR-302a-3p
miR-302b-3p

MiR $302 c-3 p$

MiR-340-5p
miR-372-3p

miR-372-3p
miR-373-3p

miR-374a-5p

miR-381-3p

MiR-410-3p
miR-424-5p

miR-449a

miR-449b-5p

niR-454-3p

nir-497-5p

miR-511-5p

miR-513b-5p

miR-519c-3p
miR-519d-3p

miR-520d-3p

miR-520e

miR-524-5p

miR- 543

niR-545-3p

niR-548c-3

miR-548e-3p

miR-590-5p

miR-590-5p
miR-607

MiR-607

miR-656-3p

miR-875-3p

HHV-6A HHV-6B HHV-7

\begin{tabular}{c|cc}
$-1,5607$ & $-2,5947$ & $-2,1$ \\
\hline 13306 & $-1,8358$ & $-1,1815$ \\
-19884 & $-2,492$ & $-1,0834$
\end{tabular}

\begin{tabular}{l|l|l|}
$-1,9484$ & $-2,4842$ & $-1,0834$ \\
\hline
\end{tabular}

\begin{tabular}{|r|r|r|}
$-1,4712$ & $-2,2066$ & $-1,471$ \\
\hline 1,2624 & $-1,2228$ & 1,2329 \\
\hline
\end{tabular}

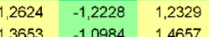

\begin{tabular}{l|l|l|}
\hline 1,3653 & $-1,0984$ & 1,4657 \\
$-1,1764$ & $-2,3201$ & 1,0647 \\
\hline
\end{tabular}

\begin{tabular}{lll}
$-1,1403$ & $-1,3949$ & $-1,6043$ \\
\hline
\end{tabular}

\begin{tabular}{|l|r|r|}
$-1,1363$ & $-2,0388$ & $-1,0489$ \\
\hline 1,201 & $-1,1867$ & 1,3663 \\
\hline
\end{tabular}

\begin{tabular}{rrr}
$-1,201$ & $-1,1867$ & 1,3663 \\
$-1,4176$ & $-1,7009$ & $-1,1413$ \\
\hline 1,107 & $-1,651$ & $-1,4039$
\end{tabular}

$\begin{array}{lll}-1,1107 & -1,6851 & -1,4039\end{array}$

\begin{tabular}{lll}
$-1,1661$ & $-1,1861$ & $-1,2382$ \\
\hline
\end{tabular}

\begin{tabular}{l|ll}
$-1,486$ & $-2,2384$ & $-2,0745$ \\
\hline
\end{tabular}

$\begin{array}{ccc}-1,5616 & -1,73 & -1,5878 \\ 1,147 & -1,2892 & 1,0074\end{array}$

\begin{tabular}{rrr}
$-1,147$ & $-1,2892$ & $-1,0074$ \\
\hline$-1,1694$ & $-1,5585$ & $-1,2069$
\end{tabular}

\begin{tabular}{rr|r|r|}
\hline$-1,1694$ & $-1,5505$ & $-1,2869$ \\
\hline$-1,0606$ & $-1,4466$ & 1,2451 \\
\hline
\end{tabular}

\begin{tabular}{llll}
$-1,0017$ & $-1,4466$ & $-1,2451$ \\
\hline & $-1,4897$ & 1,0021
\end{tabular}

\begin{tabular}{lll|}
\hline 1,0017 & $-1,4697$ & 1,0021 \\
\hline 1,1468 & $-1,3559$ & 1,4439 \\
\hline
\end{tabular}

\begin{tabular}{llll}
\hline 1,7468 & $-1,3559$ & 1,4439 \\
\hline & 1,1344 & $-1,2921$ \\
\hline
\end{tabular}

$\begin{array}{llll}1,0149 & -1,1344 & -1,2921 \\ -1,269 & -1,647 & -1,0503\end{array}$

$\begin{array}{rrr}1,0149 & 1,1344 & -1,2921 \\ -1,269 & -1,647 & -1,0603 \\ -1,0031 & -1,3018 & -1,3905\end{array}$

$\begin{array}{lll}-1,0031 & -1,3618 & -1,3986\end{array}$

$\begin{array}{rrr}-1,0991 & -1,6847 & -1,6236 \\ -1,2154 & -1,4746 & -1,1276 \\ -1,0701 & -1,4488 & -1,3083\end{array}$

$\begin{array}{rrr}-1,2154 & -1,4746 & -1,1276 \\ -1,0701 & -1,4488 & -1,3083\end{array}$

\begin{tabular}{|c|c|c|}
\hline 1,2076 & $-1,4488$ & $-1,3083$ \\
\hline 1,0334 & 1,198 \\
\hline
\end{tabular}

$\begin{array}{lll}-1,0065 & -1,3548 & -1,2124 \\ -1,0242 & -1,3965 & -1,2247\end{array}$

$\begin{array}{lll}-1,0242 & -1,3965 & -1,2247\end{array}$

\begin{tabular}{|c|c|c|}
\hline$-1,2867$ & $-2,0528$ & -2.5 \\
\hline 1,1091 & $-1,0502$ & 1,3335 \\
\hline
\end{tabular}

\begin{tabular}{llll}
1,1091 & 1,1863 & 1,9 \\
\hline
\end{tabular}

\begin{tabular}{l|l|l|}
1,1407 & $-1,2481$ & $-1,0224$ \\
\hline
\end{tabular}

\begin{tabular}{|l|l|l|}
1.0504 & $-1,5951$ & 1.0957 \\
\hline
\end{tabular}

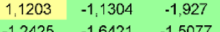

$\begin{array}{rrr}1,2425 & -1,6421 & -1,5077 \\ 1,0479 & -1,2198 & 1,1945\end{array}$

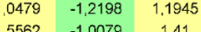

\begin{tabular}{|c|c|c|}
\hline 1,5562 & $-1,0079$ & 1,41 \\
\hline$-1,2787$ & 1,0114 & $-1,2205$ \\
\hline 1,0409 & 1,0091 & $-1,6890$
\end{tabular}

\begin{tabular}{llll}
1,0409 & 1,0091 & $-1,6889$ \\
\hline
\end{tabular}

\begin{tabular}{|c|c|c|}
\hline$-1,4808$ & $-1,4484$ & 1,9101 \\
\hline 1,1091 & $-1,4258$ & 1,3035 \\
\hline
\end{tabular}

\begin{tabular}{|c|c|c|}
\hline 1,1091 & $-1,4258$ & 1,3335 \\
\hline 13657 & 1,0819 & $-1,0345$
\end{tabular}

\begin{tabular}{|r|r|r|}
\hline$-1,0542$ & 1,0151 & 1,4111 \\
\hline 1,0199 & $-1,3714$ & 1,2307
\end{tabular}

\begin{tabular}{r|r|r}
\hline 1,0199 & $-1,3714$ & 1,2111 \\
\hline$-1,6307$
\end{tabular}

\begin{tabular}{lll}
$-1,6715$ & $-1,2947$ & $-1,8913$ \\
\hline
\end{tabular}

\begin{tabular}{ll|l|}
$-2,2345$ & $-2,4331$ & 1,1474 \\
\hline 1,4843 & $-1,6045$ & $-1,8256$
\end{tabular}

$\begin{array}{rrr}1,4843 & -1,6045 & -1,8256 \\ -1,3399 & -1,8129 & -1,5503 \\ -1,571 & -1,9217 & -1,3502\end{array}$

$\begin{array}{rrr}-1,3399 & -1,8129 & -1,5503 \\ -1,5711 & -1,9217 & -1,3502\end{array}$

$\begin{array}{rrr}-1,5711 & -1,9217 & -1,3502 \\ -1,9621 & -1,3629 & -1,9\end{array}$

\begin{tabular}{|c|c|c|}
$-1,9621$ & $-1,3629$ & $-1,9$ \\
\hline$-1,2004$ & 1,4939 & $-1,4618$ \\
\hline$-1,13$ & $-2,1255$ & $-2,197$
\end{tabular}

\begin{tabular}{l|l|l}
$-1,2004$ & 1,4939 & $-1,4618$ \\
\hline$-1,613$ & $-2,1255$ & $-2,3197$ \\
\hline$-1,2294$ & 1,2076 & $-2,1299$
\end{tabular}

\begin{tabular}{c|c|c|}
$-1,613$ & $-2,1255$ & $-2,3197$ \\
\hline$-1,2294$ & 1,2076 & $-2,1299$ \\
\hline
\end{tabular}

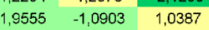

\begin{tabular}{l|l|l|l|}
$-1,1772$ & $-1,7529$ & 1,0214 \\
\hline
\end{tabular}

\begin{tabular}{|l|l|l|}
\hline 2,2472 & $-1,8445$ & 2,6387 \\
$-2,151$ & $-2,4765$ & $-1,9235$ \\
\hline
\end{tabular}

\begin{tabular}{ccc|c}
$-2,151$ & $-2,4765$ & $-1,9235$ \\
$-2,2958$ & $-2,2$ & $-2,3101$
\end{tabular}

\begin{tabular}{ll|l|l|}
1,1634 & $-2,4341$ & $-1,4356$ \\
$-1,6467$ & $-1,9873$ & $-1,2604$
\end{tabular}

$\begin{array}{rrr}-1,6467 & -1,9873 & -1,2604 \\ -1,6793 & -1,9378 & -1,7849 \\ -1,7015 & -1,8329 & -1,619\end{array}$

$\begin{array}{rrr}-1,6793 & -1,9378 & -1,7849 \\ -1,7015 & -1,8329 & -1,6199 \\ -1,0711 & -1,4909 & -1,2389\end{array}$

\begin{tabular}{lll}
$-1,0711$ & $-1,4906$ & $-1,2386$ \\
\hline
\end{tabular}

\begin{tabular}{l|l|l|l|}
$-1,4792$ & $-2,138$ & $-1,4563$ \\
\hline
\end{tabular}

$\begin{array}{lll}-1,0488 & -2,2767 & -1,042 \\ -1,0612 & -1,7601 & -1,0895\end{array}$

$\begin{array}{rrr}-1,6612 & -1,7601 & -1,0896 \\ 1,2643 & -1,7343 & -1,3935\end{array}$

\begin{tabular}{lll}
$-1,2643$ & $-1,7343$ & $-1,3935$ \\
\hline-14749 & 1,0112 & 2,0518
\end{tabular}

\begin{tabular}{r|r|r|}
\hline$-1,4749$ & 1,6112 & 2,0518 \\
1,0098 & $-1,0895$ & 1,2141 \\
\hline 1,1091 & $-1,038$ & 1,335
\end{tabular}

\begin{tabular}{ll|l}
1,1091 & $-1,038$ & 1,3335 \\
\hline
\end{tabular}

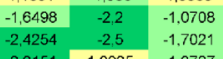

\begin{tabular}{l|c|c|}
$-2,4254$ & -2.5 & $-1,7021$ \\
$-2,2151$ & 1,0925 & $-1,3787$ \\
\hline$-1,4036$ & $-1,7051$ & $-1,678$
\end{tabular}

$\begin{array}{lll}-1,4036 & -1,7061 & -1,678 \\ -1,2903 & -1,3765 & -1,37 \\ -1,4938 & 1,2603 & -1,5411\end{array}$

\begin{tabular}{|c|c|c|}
$-1,2903$ & $-1,3765$ & $-1,37$ \\
$-1,4968$ & 1,2603 & $-1,5411$ \\
$-1,108$ & $-1,0931$ & $-1,4875$
\end{tabular}

$\begin{array}{lll}-1,108 & -1,0931 & -1,4875\end{array}$

$\begin{array}{lll}-1,2445 & -1,0846 & -1,051 \\ -1,5684 & -1,7932 & -1,2416 \\ -1,0367 & -1,901 & -1,505\end{array}$

$\begin{array}{lll}-1,5684 & -1,7932 & -1,2416 \\ -1,0367 & -1,901 & -1,505 \\ -1,2667 & -23 & -1,2561\end{array}$

\begin{tabular}{l|l|l}
$-1,0367$ & $-1,901$ & $-1,505$ \\
$-1,2667$ & -23 & $-1,2561$
\end{tabular}

$\begin{array}{lll}-1,2512 & -1,9001 & -1,488 \\ -1,2351 & -1,9624 & -1,1813\end{array}$

\begin{tabular}{ccc}
$-1,23136$ & $-2,3$ & $-1,4973$ \\
\hline 1,1091 & 1,3680 & 1,3335
\end{tabular}

\section{T lymphocytes}

\section{b}

\begin{tabular}{l|lll}
$\operatorname{miR}$ & HHV-6A & HHV-6B & HHV-7 \\
\hline & $-10-79-5 p$ & $-1,125$ & 1
\end{tabular}

\begin{tabular}{llll}
\hline let-7a-5p & $-1,0882$ & $-1,0512$ \\
\hline let-7b-5p & $-1,6773$ & 1,1955 \\
\hline let-7c-5p & 1,0305 & 1,7214
\end{tabular}

\begin{tabular}{l|c|c|}
\hline let-7c-5p & $-1,6773$ & 1,1955 \\
\hline & 1,0305 & 1,7214
\end{tabular}

let-7d-5p

let-7e-5p

let-7f-5p

$10+7 p^{2}$

let-71-5p

$\begin{array}{llll}\text { let-71-5p } & 1,3812 & 1,0437 & \mathbf{1} \\ \text { miR-9-5p } & 1,1806 & 1,035 & 1,1\end{array}$

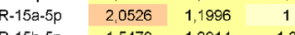

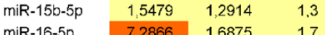

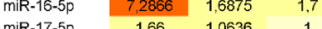

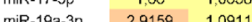

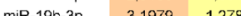

mR-1, $1,1079-1,2783$

$\begin{array}{lll}1 & 1\end{array}$

$\begin{array}{llll}\operatorname{miR}-20 D-5 p & 1,6006 & 1,1305 & 1,1 \\ \operatorname{miR}-21-5 p & 1,5017 & 1,0722 & 1\end{array}$

miR-23a-3p $1,0050 \quad 1,0203$

$\begin{array}{llll}m i R-23 b-3 p & 1,0593 & 1,4233 & 1\end{array}$

$\begin{array}{llll}m i R-29 a-3 p & 3,0687 & 1,4003 & 1,5\end{array}$

\begin{tabular}{llll} 
MiR-29o-3p & 3,6704 & 1,2013 & 1,5 \\
\hline MiR-2903p & 27142 & 1,2038 & 1,
\end{tabular}

$\begin{array}{llll}m, R-29 C-3 p & 2,7142 & 1,2038 & 1,6\end{array}$

$\begin{array}{llll}\text { miR-30-5p } 5 p & 1,9954 & 1,1417 & 1\end{array}$

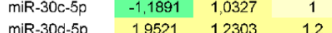

$\begin{array}{llll}\text { miR-30d-5p } & 1,9521 & 1,2303 & 1,2\end{array}$

\begin{tabular}{|l|l|l|l|}
\hline miR-30e-5p & 2,3324 & 1,1245 & 1 \\
\hline miR-34a-5p & 55154 & $-1,2654$ & -2 \\
\hline
\end{tabular}

\begin{tabular}{l|l|l} 
MiR-34a-5p & 5,5154 & $-1,2654$ \\
\hline MiR-34c-5p & 29197 & 1,0296
\end{tabular}

$\begin{array}{lll}\text { miR-39-3p } & 2,9197 \quad 1,0296\end{array}$

\begin{tabular}{lll} 
miR-39-3p $\quad 2,9197$ & 1,0296 \\
\hline
\end{tabular}

\begin{tabular}{l|l|l|l} 
miR-93-5p & $-1,0018$ & 1,0132 & 1.3 \\
\hline
\end{tabular}

\begin{tabular}{l|l|l|l|}
\hline miR-98-5p & 1,0821 & $-1,0128$ & -1 \\
\hline M & 3,1037 & 13258 & 1,4
\end{tabular}

\begin{tabular}{l|l|l|l}
\hline miR-101-3p & 3,1937 & 1,3258 & 1.4 \\
\hline mR-105b-5p & 2,1782 & 1,1897 & 1.2
\end{tabular}

$\begin{array}{llll}m i R-125 a-50 & -1,3316 & 1,0888\end{array}$

$\begin{array}{llll}\text { miR-125b-5p } & 3,4296 & 1,0296 & 1\end{array}$

\begin{tabular}{l|c|c|c}
\hline $\operatorname{miR}-128-3 p$ & 2,309 & 1,1265 & 1,1 \\
\hline
\end{tabular}

\begin{tabular}{l|l|l|l} 
miR-130b-3p & 3,4965 & 1,1658 & 1,1
\end{tabular}

\begin{tabular}{llll} 
miR-144-3p & 2,9538 & 1,0296 & 1 \\
\hline
\end{tabular}

\begin{tabular}{l|l|l|l|}
\hline miR-145-5p & 5,3457 & $-2,386$ & $-1,5$ \\
\hline
\end{tabular}

$\begin{array}{llll}M R-1810-50 & -1,1456 & 1,0043 & 1\end{array}$

$\begin{array}{lll}\text { miR-181c-5p } 1,468 & -1,0050 & -1\end{array}$

\begin{tabular}{|l|l|l|l|}
\hline miR-181d-5p & $-3,0667$ & 1,2042 & 1.4
\end{tabular}

$\begin{array}{lllll}\text { miR-186-5p } & 2,0493 & 1,0671 & 1\end{array}$

$\begin{array}{llll}\text { miR-195-5p } & 1,5083 & 1,1593 & 1,2\end{array}$

\begin{tabular}{l|l|l|l|l|l|l|l|l|l|l|l|} 
miR-195-5p & 1,5083 & 1,1593 & 1,2 \\
miR-202-3p & 11,3395 & 1,0296 & 1
\end{tabular}

$\begin{array}{llll}\text { miR-211-5p } & 2,9197 & 1,0296 & 1\end{array}$

\begin{tabular}{l|c|cc}
$\operatorname{miR}-300$ & 2,9197 & 1,0296 & 1.2 \\
\hline $\operatorname{miR}-301 \mathrm{a}-30$ & 6.9893 & 1.0421 & 1
\end{tabular}

\begin{tabular}{c|c|c|c}
\hline $\operatorname{miR}-301 a-3 \rho$ & 6,9893 & 1,0421 & 1 \\
\hline $\operatorname{miR}-301 \mathrm{~b}-30$ & 10.2386 & 3,871 & 2.5 \\
\hline
\end{tabular}

\begin{tabular}{l|l|l|l|} 
miR-302a-3p & 2,9197 & 1,0296 & $\mathbf{1}$
\end{tabular}

$\begin{array}{lll}\text { miR-302b-3p } \quad 2,9197 & 1,0296\end{array}$

\begin{tabular}{lll} 
miR-302c-3p & $39,2 / 26$ & 1,0296 \\
\hline
\end{tabular}

\begin{tabular}{lll} 
miR-340-5p & 1,4727 & $-1,1196$ \\
\hline
\end{tabular}

$\begin{array}{lll}\operatorname{miR}-372-3 p & 3,3602 & 1,0296\end{array}$

$\begin{array}{lll}\text { miR-373-3p } & 2,9197 & 1,0296\end{array}$

miR-374a-5p $1,7526 \quad 1,1065$

miR-381-3p $7,641 \quad 1,0296$

$\begin{array}{lll}\operatorname{miR}-410-3 p & 2,9599 & 1,0296\end{array}$

\begin{tabular}{lll|l} 
miR-424-5p & 5,0353 & 1,2494 \\
\hline
\end{tabular}

$\begin{array}{lll}\operatorname{miR}-449 a & 2,9197 \quad 1,029\end{array}$

\begin{tabular}{l|l|l|l} 
miR-449b-5p & 14,3673 & 1,0296 \\
\hline
\end{tabular}

$\begin{array}{lll}\text { miR-454-3p } & -1,5806 & 1,1155\end{array}$

$\begin{array}{lll}\text { miR-497-5p } & 4,3364 & 1,2219\end{array}$

\begin{tabular}{l|l|l} 
MiR-511-5p & 2,9197 & 1,0296 \\
\hline
\end{tabular}

\begin{tabular}{l|l|l} 
miR-513b-5p & 2,9197 & 1,0296 \\
\hline
\end{tabular}

\begin{tabular}{lll} 
miR-519c-3p & 2,9197 & 1,0296 \\
\hline
\end{tabular}

\begin{tabular}{lll} 
miR-519d-3p & 2,9197 & 1,0296 \\
\hline
\end{tabular}

$\begin{array}{lll}\text { miR-520d-3p } & 3,4069 & 1,0296\end{array}$

\begin{tabular}{lll} 
miR-520e & 2,9197 & 1,0296 \\
\hline
\end{tabular}

$\begin{array}{lll}R-524-5 p & 4,5085 & 1,0296\end{array}$

\begin{tabular}{llll}
\hline$R-543$ & 2,9197 & 1,029 \\
\hline$M R-545-3 p$ & 29197 & 1,0296
\end{tabular}

MiR. 548c 3p $29197 \quad 1,020$

\begin{tabular}{lllr} 
MiR-548c-3p & 2,6301 & 1,0296 & 1 \\
\hline MR-585 & 1,3
\end{tabular}

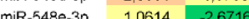

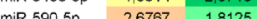

\begin{tabular}{lll}
$M R-607$ & 2,6767 & $-1,8125$ \\
\hline miR-607 & 2,9197 & 1,0296
\end{tabular}

R-656-3

miR-875-3

\begin{tabular}{r|r}
2,9197 & 1,0296 \\
2,9197 & 1,0296
\end{tabular}

$2,9197 \quad 1,0296$

\begin{tabular}{r|r}
9197 & 1,0296 \\
\hline
\end{tabular}

Fig. 2 Herpesviruses-induced modulation of autoimmunity-related miRNAs in thyroid and T lymphoid cell lines. Nthy-ori3-1 and Jurkat T cells were uninfected (control) or infected with HHV-6A, HHV-6B or HHV-7 and analysed at 48 h.p.i. for miRNA expression by a microarray analysis evidencing 84 miRNAs associated to inflammation and autoimmunity. Results are expressed as fold-change compared to control values, and represent the mean value of triplicate samples. a miRNA expression in thyroid cells $\mathbf{b}$ miRNA expression in T lymphocytes 

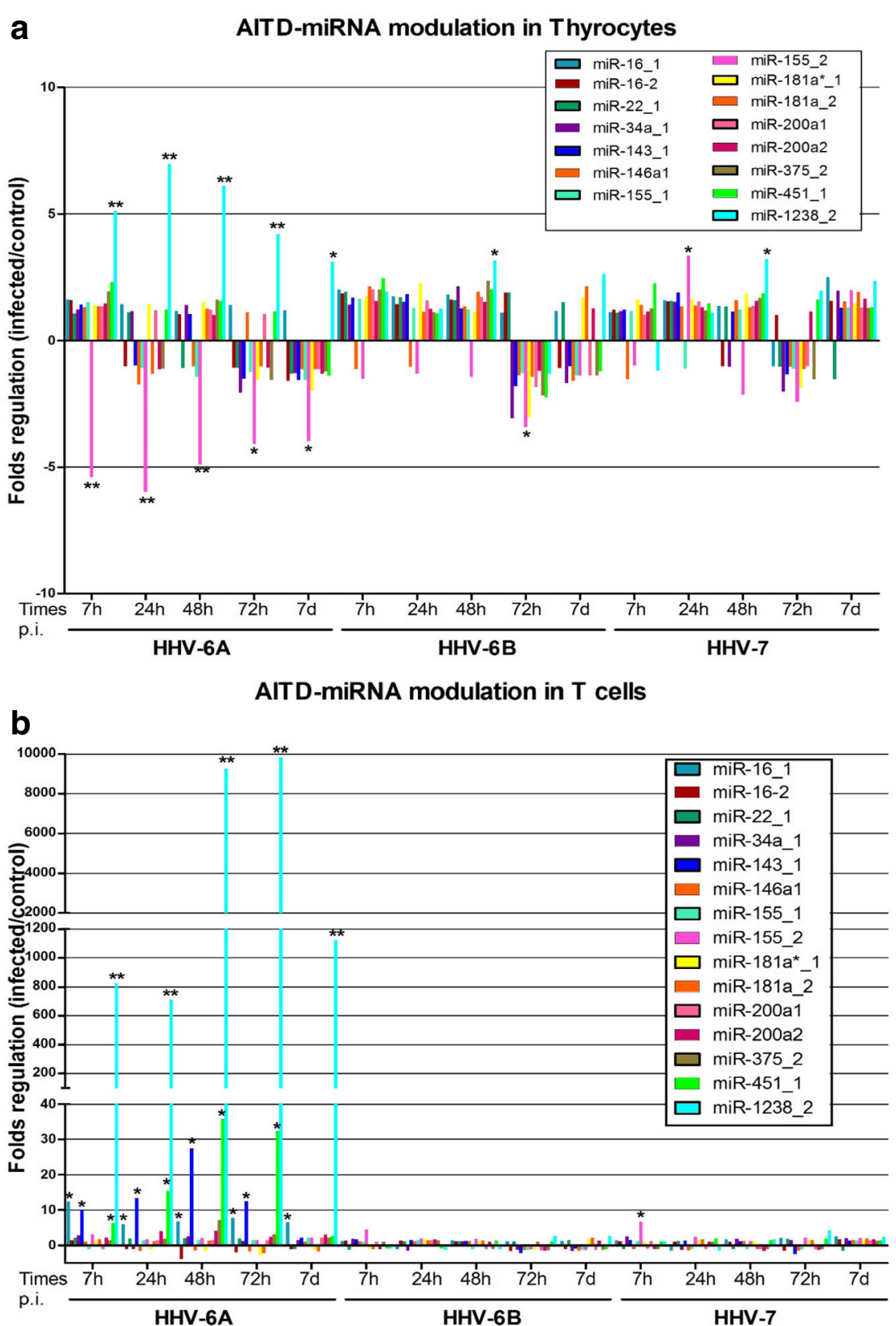

Fig. 3 Herpesviruses-induced modulation of AITD-related miRNAs in thyroid and T lymphoid cell lines. Nthy-ori3-1 and Jurkat T cells were uninfected (control) or infected with HHV-6A, HHV-6B or HHV-7 and analysed for expression of individual AITD-associated miRNAs at the indicated times post infection. Results are expressed as fold-change compared to control values, and represent the mean value of triplicate samples in two independent experiments. a miRNA expression in thyroid cells $\left({ }^{*}, p<0.01 ;{ }^{*}, p<0.001\right)$. b miRNA expression in T lymphocytes $\left({ }^{*}, p<0.001\right.$; **, $p<0.0001$ )

infected cells, further strengthening the notion that $6 \mathrm{~A}$ and $6 \mathrm{~B}$ species induce diverse effects inside infected cells, and are associated to diverse diseases. Since all used HHV show superimposable structural features and ability to enter and replicate in infected cells, the observed differences might be attributable to factors induced or expressed differently by these viruses inside infected cells. These factors may include both viral and virus-induced cell compounds, which will be interesting to analyze in future studies.

Overall, our current findings add evidences supporting the association between HHV-6A infection and AITD onset, and suggest a potential role of the induced miRNA in the development ofHHV-6A associated diseases. Further studies are however needed to verify these effects in primary human cells and to assess the 
functional role of virus-induced miRNAs in the development of the virus-induced autoimmunity.

\section{Acknowledgements}

We thank Iva Pivanti and Erica Gentilin for their excellent technical assistance.

\section{Funding}

This work was partially supported by Funds for Basic Research of the University of Ferrara.

\section{Availability of data and materials}

The datasets during and/or analysed during the current study available from the corresponding author on reasonable request.

\section{Authors' contributions}

EC conceived and designed the experiments; EC, MD, IS performed the experiments; MCZ, RR, EU analysed and discussed the data; EC and DDL wrote the paper. All authors read and approved the final manuscript.

\section{Competing interests}

The authors declare that they have no competing interests.

\section{Consent for publication}

Not required.

\section{Ethics approval and consent to participate}

Not required.

\section{Author details}

${ }^{1}$ Department of Medical Sciences, Section of Microbiology and Medical Genetics, University of Ferrara, via L. Borsari 46, 44121 Ferrara, Italy. ${ }^{2}$ Department of Medical Sciences, Section of Endocrinology and Internal Medicine, University of Ferrara, via A. Moro 8, 44124 Ferrara, Italy. ${ }^{3}$ Endocrinology Unit, Azienda Ospedaliero-Universitaria di Ferrara, via A. Moro 8, 44124 Ferrara, Italy.

\section{Received: 13 October 2016 Accepted: 15 December 2016}

Published online: 11 January 2017

\section{References}

1. McLeod DS, Cooper DS. The incidence and prevalence of thyroid autoimmunity. Endocrine. 2012:42:252-65.

2. Thomas D, Liakos V, Michou V, Kapranos N, Kaltsas G, Tsilivakos V, Tsatsoulis A. Detection of herpes virus DNA in post-operative thyroid tissue specimens of patients with autoimmune thyroid disease. Exp Clin Endocrinol Diabetes. 2008;116:35-9

3. Caselli E, Zatelli MC, Rizzo R, Benedetti S, Martorelli D, Trasforini G, Cassai E, Degli Uberti EC, Di Luca D, Dolcetti R. Virologic and immunologic evidence supporting an association between HHV-6 and Hashimoto's thyroiditis. PLoS Pathog. 2012;8:e1002951.

4. Rizzo R, Zatelli MC, Rotola A, Cassai E, Degli Uberti E, Di Luca D, Caselli E: Increase in Peripheral CD3-CD56brightCD16- Natural Killer Cells in Hashimoto's Thyroiditis Associated with HHV-6 Infection. Adv Exp Med Biol. 2015;897:113-20.

5. Caselli E, Di Luca D. Molecular biology and clinical associations of Roseoloviruses human herpesvirus 6 and human herpesvirus 7. New Microbiol. 2007:30:173-87.

6. Ablashi D, Agut H, Alvarez-Lafuente R, Clark DA, Dewhurst S, DiLuca D, Flamand L, Frenkel N, Gallo R, Gompels UA, et al. Classification of HHV-6A and HHV-6B as distinct viruses. Arch Virol. 2014;159:863-70

7. Tang H, Wang J, Mahmoud NF, Mori Y. Detailed study of the interaction between human herpesvirus 6B glycoprotein complex and its cellular receptor, human CD134. J Virol. 2014;88:10875-82.

8. Jasirwan C, Furusawa Y, Tang H, Maeki T, Mori Y. Human herpesvirus-6A gQ1 and gQ2 are critical for human CD46 usage. Microbiol Immunol. 2014; 58:22-30.

9. Broccolo F, Fusetti L, Ceccherini-Nelli L. Possible role of human herpesvirus 6 as a trigger of autoimmune disease. Scientific World Journal. 2013;2013: 867389.
10. Bernecker C, Lenz L, Ostapczuk MS, Schinner S, Willenberg H, Ehlers M, Vordenbaumen S, Feldkamp J, Schott M. MicroRNAs miR-146a1, miR-155_2, and miR-200a1 are regulated in autoimmune thyroid diseases. Thyroid. 2012;22:1294-5.

11. Yamada H, Itoh M, Hiratsuka I, Hashimoto S. Circulating microRNAs in autoimmune thyroid diseases. Clin Endocrinol (Oxf). 2014:81:276-81.

12. Xiao C, Rajewsky K. MicroRNA control in the immune system: basic principles. Cell. 2009;136:26-36.

13. Caselli E, Bracci A, Galvan M, Boni M, Rotola A, Bergamini C, Cermelli C, Dal Monte P, Gompels UA, Cassai E, Di Luca D. Human herpesvirus 6 (HHV-6) U94/REP protein inhibits betaherpesvirus replication. Virology. 2006;346:402-14

14. Baltimore D, Boldin MP, O'Connell RM, Rao DS, Taganov KD. MicroRNAs: new regulators of immune cell development and function. Nat Immunol. 2008;9:839-45.

15. Rodriguez A, Vigorito E, Clare $S$, Warren MV, Couttet $P$, Soond DR, van Dongen S, Grocock RJ, Das PP, Miska EA, et al. Requirement of bic/ microRNA-155 for normal immune function. Science. 2007;316:608-11.

16. Sullivan RP, Fogel LA, Leong JW, Schneider SE, Wong R, Romee R, Thai TH, SexI V, Matkovich SJ, Dorn 2nd GW, et al. MicroRNA-155 tunes both the threshold and extent of NK cell activation via targeting of multiple signaling pathways. J Immunol. 2013;191:5904-13.

17. Gracias DT, Stelekati E, Hope JL, Boesteanu AC, Doering TA, Norton J, Mueller YM, Fraietta JA, Wherry EJ, Turner M, Katsikis PD. The microRNA miR-155 controls CD8(+) T cell responses by regulating interferon signaling. Nat Immunol. 2013;14:593-602

18. O'Connell RM, Kahn D, Gibson WS, Round JL, Scholz RL, Chaudhuri AA, Kahn ME, Rao DS, Baltimore D. MicroRNA-155 promotes autoimmune inflammation by enhancing inflammatory T cell development. Immunity. 2010;33:607-19.

19. Pekarsky Y, Croce CM. Role of miR-15/16 in CLL. Cell Death Differ. 2015;22: 6-11.

20. Leong JW, Sullivan RP, Fehniger TA. microRNA management of NK-cell developmental and functional programs. Eur J Immunol. 2014;44:2862-8.

21. Jia X, Li X, Shen Y, Miao J, Liu H, Li G, Wang Z. MiR-16 regulates mouse peritoneal macrophage polarization and affects T-cell activation. J Cell Mo Med. 2016:20:1898-907.

22. Shi X, Zhan L, Xiao C, Lei Z, Yang H, Wang L, Zhao J, Zhang HT. miR-1238 inhibits cell proliferation by targeting LHX2 in non-small cell lung cancer. Oncotarget. 2015;6:19043-54.

23. Glinsky GV. An SNP-guided microRNA map of fifteen common human disorders identifies a consensus disease phenocode aiming at principal components of the nuclear import pathway. Cell Cycle. 2008;7:2570-83.

\section{Submit your next manuscript to BioMed Central} and we will help you at every step:

- We accept pre-submission inquiries

- Our selector tool helps you to find the most relevant journal

- We provide round the clock customer support

- Convenient online submission

- Thorough peer review

- Inclusion in PubMed and all major indexing services

- Maximum visibility for your research

Submit your manuscript at www.biomedcentral.com/submit
Biomed Central 